Military Technical College Kobry El-Kobbah, Cairo, Egypt.

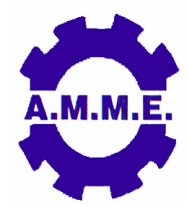

$15^{\text {th }}$ International Conference on Applied Mechanics and Mechanical Engineering.

\title{
DESIGN OPTIMIZATION OF THIN WALLED STRUCTURES UNDER IMPACT LOADING USING THE RESPONSE SURFACE METHOD
}

\author{
H. M. Eltaher *
}

\begin{abstract}
Structural design optimization under impact loading faces two difficulties. First, the inherent complexity of the finite element model required for modeling the complicated physical phenomenon, and second the high computational cost required by the iterative nature of numerical optimization. This paper presents a practical approach for solving this problem by using the response surface method as an approximate model rather than the computationally expensive one. Then numerical optimization can be conducted at an affordable computational cost. Finally, only one simulation analysis is conducted using the initial finite element model to verify the optimum design achieved from numerical optimization. The proposed approach has been successfully applied to the problem of designing a thin walled tube under impact loading which suggests that this approach can be used for solving design optimization problems of other complex structures.
\end{abstract}

\section{KEY WORDS}

Crashworthiness; Vehicle design; Optimization; LS-DYNA; Automobile structures; Approximate methods; RSM; DOE

\footnotetext{
* Egyptian Armed Forces
} 


\section{INTRODUCTION}

Structures under impact loading go through large deformations and stresses exceed materials elastic limits into plastic regions. Furthermore, parts are pressed against each others under the large forces of impact. This produces contact forces and friction between these parts. The whole incident occurs during very short time (about $100 \mathrm{~ms}$ ). Considering this, the nonlinear finite element (FE) method required for modeling is highly sophisticated and demands huge calculations. For example, a simulation of full frontal impact of a full vehicle model may last for more than a half day [1].

Optimization is a numerical technique that systematically and automatically searches the design space through numerous iterations to find an optimum feasible solution [2]. Since, each analysis is computationally expensive; hence conducting optimization directly to nonlinear finite element models can be prohibitively computationally expensive.

This paper presents a practical approach for solving this problem by using the response surface method as an approximate model instead of the computationally expensive finite element model. Then numerical optimization can be conducted at an affordable computational cost. Finally, only one simulation analysis is conducted using the initial finite element model to verify the optimum design achieved from numerical optimization. The proposed approach is illustrated in Fig. 1.

\section{RESPONSE SURFACE METHOD (RSM)}

RSM is a global approximation technique which can be used to construct simple and easy to calculate surrogate models of the highly complex nonlinear FE models. The output models are in the form of smooth polynomial functions describing the output response in terms of the input design variables. Thus the noisy behaviour of output responses which typically encountered in crash analysis problems can be easily alleviated. These polynomials can then be used in evaluating the system response within the design space at an affordable computational cost. However, to create these approximate models, several evaluations are required to identify the unknown polynomial coefficients. Because crashworthiness evaluations are expensive, Design of Experiments (DOE) is used to minimize the number of points at which simulation will take place. Simulations are then performed at these strategic points, and data is processed by regression analysis to identify the unknown polynomial coefficients [3]. The accuracy of the models is quantified to determine their validity to represent the system instead of the true (expensive) models. Having these models validated, they can be effectively used to perform design optimization at a much lower computational cost.

\section{Regression Analysis}

Regression analysis is used to identify the unknown polynomial coefficients in RSM. Assume $y=X \beta+\varepsilon$ where $\mathrm{y}$ is an $(n \times 1)$ vector that contains the exact responses calculated from Nonlinear FE simulations, $X$ is an $(n \times p)$ design matrix determined based on the type of choice of the polynomial functions; $\beta$ is a $(p \times 1)$ vector of the 
unknown model polynomial coefficients, and $\mathcal{E}$ is a $(n \times 1)$ vector that contains the errors between exact and approximate responses. Using least squares method [4], the coefficients can be calculated as:

$$
\beta=\left(X^{T} X\right)^{-1} X^{T} y
$$

\section{Approximate Models}

There are four types of regression models, which can be described as follows:

A linear model (Model 1) that includes only basic variables and does not include any interaction between variables which is described as:

$$
\hat{y}=a_{0}+a_{1} x_{1}+a_{2} x_{2}+a_{3} x_{3}
$$

where $\hat{y}$ is the approximated response, $a_{i}$ are the unknown coefficients of the approximate models and $x_{i}$ are the design variables, which are considered here as the thickness of each part. This type of model, represent a hyper-plane in the 3 dimension design space with no curvature since only first degree variables are included. The constant term represents the initial value of the response.

A linear model with interaction (Model 2) that includes constant, linear, and interaction terms as in the following form:

$$
\hat{y}=a_{0}+a_{1} x_{1}+a_{2} x_{2}+a_{3} x_{3}+a_{12} x_{1} x_{2}+a_{13} x_{1} x_{3}+a_{23} x_{2} x_{3}
$$

This model allows for the first order interaction between variables and it represents a hyper-plane in the variables space.

A quadratic model (Model 3) that includes constant, linear, interaction, and squared terms in the following form:

$$
\hat{y}=a_{0}+a_{1} x_{1}+a_{2} x_{2}+a_{3} x_{3}+a_{12} x_{1} x_{2}+a_{13} x_{1} x_{3}+a_{23} x_{2} x_{3}+a_{11} x_{1}^{2}+a_{22} x_{2}^{2}+a_{33} x_{3}^{2}
$$

This model is quite flexible in representing wide varieties of models especially when there is no prior knowledge about the type of the model to be used. Thus it is the default model of choice in RSM application. It represents a hyper plane in the design space with curvature due to the second degree terms.

A pure quadratic model (Model 4) that includes constant, linear, and squared terms described in the following form:

$$
\hat{y}=a_{0}+a_{1} x_{1}+a_{2} x_{2}+a_{3} x_{3}+a_{11} x_{1}^{2}+a_{22} x_{2}^{2}+a_{33} x_{3}^{2}
$$

This model represents a hyper-plane with curvature, but it includes no interacting terms.

Meta-model selection is the process in which the designer tries to find the best approximation that fits the data. A prior knowledge about the system being modeled will facilitate this process. This knowledge can include the type of variables important 
to the output response, which will help in reducing the number of variables by screening out important ones. Also, knowledge about the type and level of interaction between variables, if any exists, will help in formulating the approximate model. However, this information is not always available, and a trial and error process is inevitable. In such a case, different meta-models are evaluated based on their success in approximating the data; the model with the highest accuracy is then selected.

\section{Measuring Accuracy}

It is important that the accuracy of the developed meta-model is verified so that it can be used with confidence in the optimization process. The coefficient of determination known as $R^{2}$ is typically used to check the model's ability to identify the variation within the output response [4] and is defined as:

where

$$
R^{2}=1-\frac{S S E}{S S T}
$$

and

$$
\operatorname{SSE}=\sum_{i=1}^{n}\left(y_{i}-\hat{y}_{i}\right)^{2}
$$

$$
S S T=\sum_{i=1}^{n}\left(y_{i}-\bar{y}_{i}\right)^{2}
$$

$y_{i}$ is the true output response, here calculated from nonlinear FE analysis, $\hat{y}_{i}$ is the approximate response calculated from RSM, $\bar{y}$ is the average of the true response, and $n$ is the number of design points used to generate the model. $R^{2}$ varies between 0 and 1 , where values close to 1 mean that the approximate model has high ability to explain the variations within the output response.

It should be noted that the value of $R^{2}$ can increase by adding unnecessary variables. In fact $R^{2}$ can be brought to 1 if a model with $(n-1)$ of variables is used to fit $\mathrm{n}$ points. Therefore, adj $R^{2}$ is used instead, which adjusts $R^{2}$ by dividing both $S S E$ and $S S T$ by their associated degrees of freedom as:

$$
\operatorname{adj} R^{2}=1-\frac{\frac{S S E}{(n-p)}}{\frac{S S T}{(n-1)}}
$$

where $p$ is the number of design variables. $\operatorname{adj} R^{2}$ will then account for adding unimportant design variables. Since for $\mathrm{n}$ points, the value of $S S T /(n-1)$ is constant, then $\operatorname{adj} R^{2}$ will increase only when adding a new variable results in reducing the value of $S S E /(n-p)$. In fact $\operatorname{adj} R^{2}$ may even decrease when adding a new variable decreases the value of $(n-p)$ more than it decreases the value of SSE.

\section{DESIGN OF EXPERIMENTS}


To determine the polynomial coefficients $\beta$, simulation is conducted at various points in the design space to generate the $y$ responses. The location of these points should be chosen carefully to generate accurate models. Naturally, as the number of points covering the design space increases, the model's accuracy also increases. However, since crashworthiness simulation is computationally expensive, the number of simulations should be optimized to produce accurate models with minimum simulation runs. DOE is a statistical technique which can be used to locate the optimum number of experiments to be carried on. The D-optimality criterion is one of the most popular techniques which depends on maximizing the value of $\left|X^{T} X\right|$, as the value of the variance in $\beta, \operatorname{var}(\beta)$ is related to $\left(X^{T} X\right)$ as:

$$
\operatorname{var}(\beta) \propto\left(X^{T} X\right)^{-1}
$$

Therefore considering Eq.(10), maximizing $\left|X^{T} X\right|$ leads to less variation in $\beta$ and more accurate models.

\section{OPTIMIZATION OF A THIN WALLED TUBE}

A thin walled tube is divided into three parts as shown in Fig.1. A base design is made of steel with a constant walled thickness of $2 \mathrm{~mm}$. Parts thicknesses are assumed to be the design variables, where $\mathrm{XL}[1,1,1]$ and $\mathrm{XU}[3,3,3]$ are the lower and upper bounds on the design variables. The load is assumed as an axial impact loading from a $100 \mathrm{~kg}$ dropped mass with a $10 \mathrm{~m} / \mathrm{s}$ pre-impact speed. The load is modeled using the RIGIDWALLED PLANAR MOVING FORCES keyword in LSDYNA [5]. The tube is modeled using a nonlinear FE model and LS-DYNA is used for simulations. The tube walls are modeled with 391 Belytschko-Lin-Tsay shell elements. This element provides a balance between accuracy and computational efficiency. The tube has a $235 \mathrm{~mm}$ length and $55 \mathrm{~mm}$ edge length. The material is modeled with the MAT PIECEWISE LINEAR PLASTICITY model (model type 24 in LS-DYNA) [5]. The material parameters for steel are: a Young's modulus of 207 $\mathrm{GPa}$, a Poisson's ratio of 0.3 , a density of $7830 \mathrm{~kg} / \mathrm{m} 3$, a yield stress of $200 \mathrm{MPa}$, and a tensile strength of $448 \mathrm{MPa}$. The Cowper-Symonds material strain rate sensitivity parameters are assumed to be $\mathrm{D}=6844 \mathrm{~s}-1$ and $\mathrm{q}=3.91$ as suggested by Abrawicz and Jones [6]. The contact between the tube walls is modeled with the CONTACT AUTOMATIC SINGLE SURFACE keyword in LS-DYNA. The nonlinear FE model verified with the "symmetric crush tube" model in the examples manual in LS-DYNA software [5].

\section{RESULTS AND DISCUSSIONS}

The D-Optimality criterion is used to generate 15 points as provided in Table 1 and nonlinear FE analysis has been conducted at each point. The results of Impact Energy $(I E)$ and mass are recorded and used to fit each of the four different RSM models (linear, interaction, quadratic and pure quadratic). The value of the adjR2 is calculated for each model type and provided in Table 2. Again, the results show that 
the quadratic model is the most accurate and is used here to model both IE and mass in the optimization problem.

The optimization problem is formulated to search for the optimum thickness values that maximize IE while maintaining the same weight as the base design and the problem can be written as: Using the SQP algorithm, starting from the base design at $X=\left(\begin{array}{ll}2 & 2\end{array}\right)$, an optimum solution was found after 15 iterations at $X^{*}=\left(\begin{array}{lll}3 & 1.36 & 1.8\end{array}\right)$ and the iteration history is shown in

Fig. 3. The SQP algorithm is considered one of the most efficient numerical optimization algorithms [7]. From

Fig. 3, it is clear that the algorithm has managed to increase the value of the objective function (IE) after 15 iterations. It is also clear that the value of $I E$ has stabilized in the last 6 iterations.

The optimum design absorbs $2.75 \mathrm{~kJ}$, which is about $28 \%$ larger than that of the base design $(2.15 \mathrm{~kJ})$ while maintaining the base design mass. A comparison between the base and optimum designs are presented in

Fig. 4. It is clear that optimization has successfully improved the base design by increasing its ability to absorb impact energy $(I E)$ without increasing its weight. This has been accomplished by obtaining the optimum (best) arrangement of thickness values. It should be noted here that this accomplishment has been made practically possible by the proposed approach which required only minimum number of the computationally expensive nonlinear FE simulations.

\author{
Find $X^{*}$ that: \\ Minimizes IE \\ Subject to: Mass-Mass original $\leq 0$ \\ where, $X_{L} \leq X \leq X_{U}$
}

\title{
CONCLUSIONS
}

Optimization of thin walled structures under dynamic impact loading is a challenging engineering task. In this paper, it has been demonstrated that this complicated task can be undertaken using design of experiments and regression analysis. The creation of approximate models in the form of polynomials those can be easily computed has enabled the practical application of optimization using any numerical algorithm. The approach has succeeded in improving the design of a thin walled tube at an acceptable computational cost. It is believed that this approach can be extended for improving large scale structures.

\section{REFERENCES}

[1] Natori, S. and Q. Yu, "An Application of CAP (Computer-Aided Principle) to Structural Design for Vehicle Crash Safety", SAE Technical paper 2007-01-0882 (2007).

[2] Venkataraman, P., "Applied optimization with MATLAB programming", WileyInterscience (2002). 
[3] Myers, R.H. and D.C. Montgomery, "Response surface methodology : process and product optimization using designed experiments", Wiley (1995).

[4] Montgomery, D.C., E.A. Peck and G.G. Vining, "Introduction to linear regression analysis", J. Wiley \&amp; Sons (2006).

[5] LS-DYNA, http://www.Istc.com/lsdyna.htm. [cited 2012 February 17].

[6] Abramowicz, W. and N. Jones, "Dynamic progressive buckling of circular and square tubes", International Journal of Impact Engineering, Vol. 4, no. 4, pp. 243-270 (1986).

[7] Rao, S.S., "Engineering optimization theory and practice", (1996). 


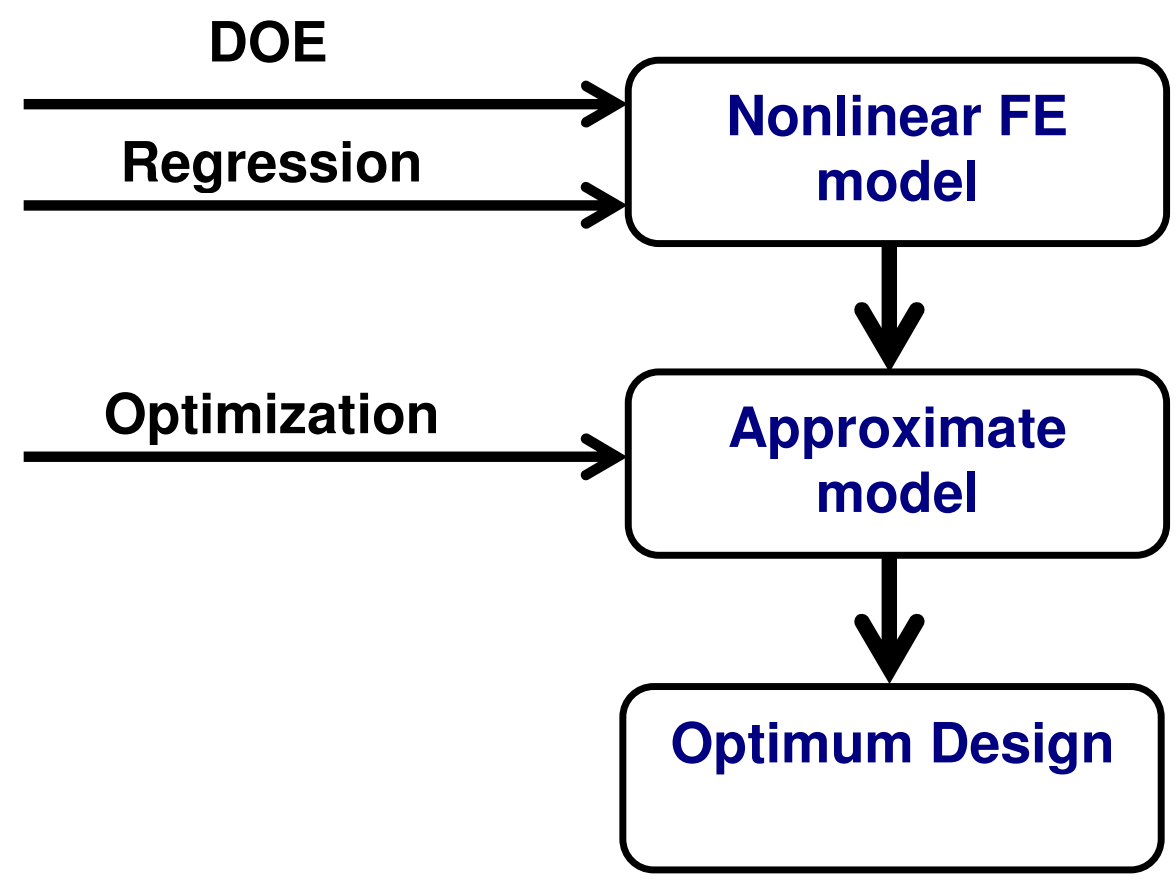

Fig.1. Schematic drawing of the proposed approach.

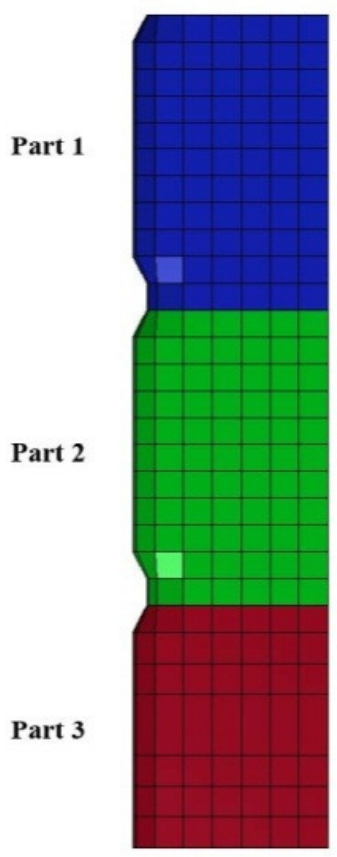

Fig. 2. A view of the finite element model of the thin walled tube. 


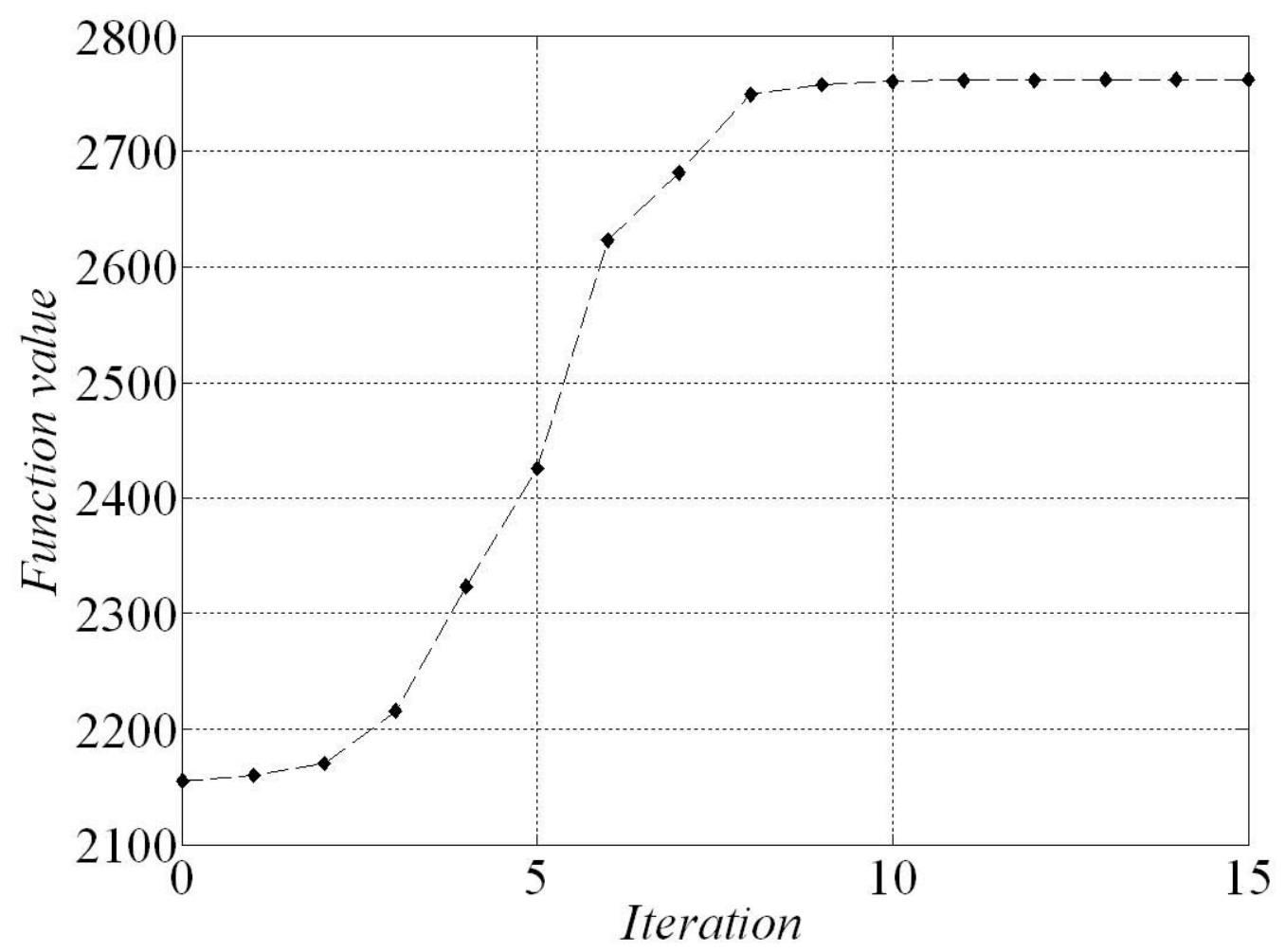

Fig. 3. Iteration history of the thickness optimization problem for thin walled.

\section{口ass $\square$ IE}

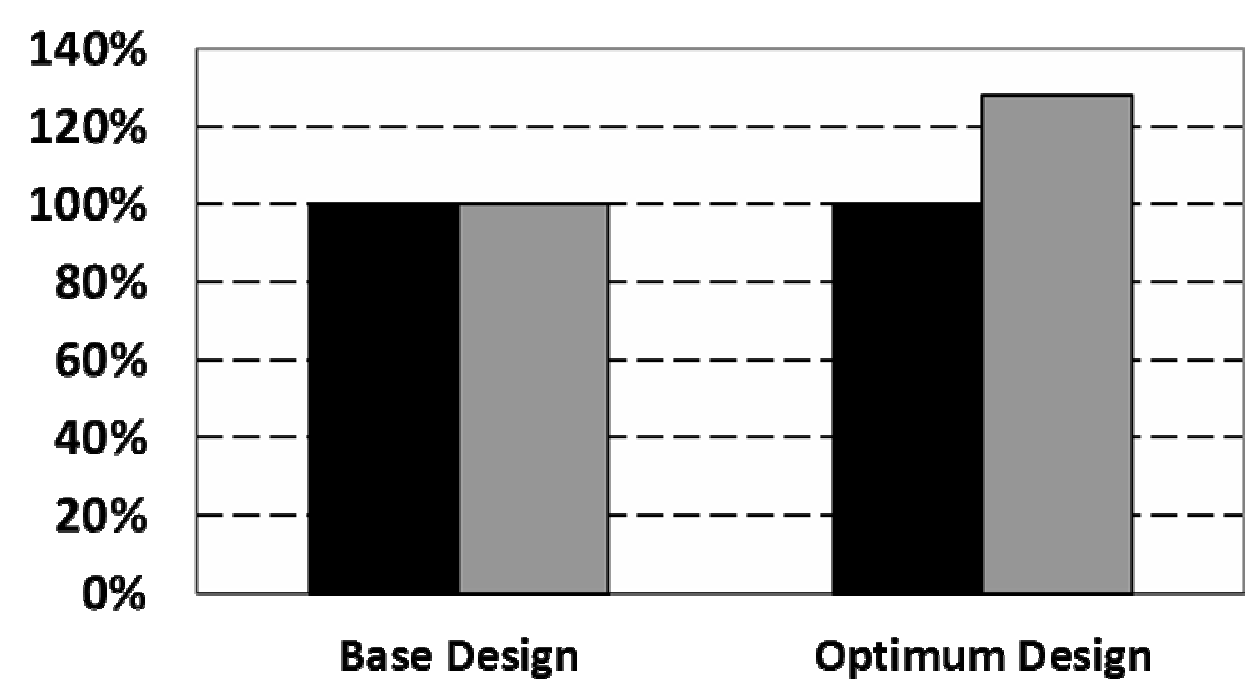

Fig. 4. Comparison between base and optimum design. 
Table 1. Design matrix of the RSM model for the thin walled tube.

\begin{tabular}{c|cccccccc}
\hline ID & $\mathbf{1}$ & $\mathbf{2}$ & $\mathbf{3}$ & $\mathbf{4}$ & $\mathbf{5}$ & $\mathbf{6}$ & $\mathbf{7}$ & $\mathbf{8}$ \\
\hline T1 & 2 & 1.74 & 2.19 & 2.6 & 2.45 & 1.54 & 2.9 & 2.5 \\
T2 & 2 & 1.79 & 2.35 & 2.4 & 1.41 & 2.97 & 2.92 & 1.8 \\
T3 & 2 & 2.13 & 2.07 & 2.09 & 1.03 & 2.11 & 2.04 & 1.09 \\
\hline ID & $\mathbf{9}$ & $\mathbf{1 0}$ & $\mathbf{1 1}$ & $\mathbf{1 2}$ & $\mathbf{1 3}$ & $\mathbf{1 4}$ & $\mathbf{1 5}$ & \\
\hline T1 & 1.23 & 2.35 & 1.93 & 1.39 & 2.93 & 2.51 & 2.12 & \\
T2 & 1.75 & 2.96 & 1.12 & 2.59 & 1.29 & 1.94 & 1.03 & \\
T3 & 1.5 & 2.23 & 2.35 & 1.48 & 2.3 & 1.92 & 1.5 & \\
\hline
\end{tabular}

Table 2. adjR2 values for the different RSM models for thin walled tube Mass and IE.

\begin{tabular}{c|cccc}
\hline & Linear & Interaction & Quadratic & Pure quadratic \\
\hline Mass & 1 & 1 & 1 & 1 \\
IE & 0.82 & 0.85 & 0.91 & 0.88 \\
\hline
\end{tabular}

